\title{
Development of Hybrid Algorithm Based on PSO and NN to Solve Economic Emission Dispatch Problem
}

\author{
R. Leena Rose ${ }^{*}$, B. Dora Arul Selvi², R. Lal Raja Singh ${ }^{3}$ \\ ${ }^{1}$ Department of Electrical and Electronics Engineering, Sasurie Academy of Engineering, Coimbatore, India \\ ${ }^{2}$ Department of Electrical and Electronics Engineering, Holycross Engineering College, Tuticorin, India \\ ${ }^{3}$ Kalaignarkarunanidhi Institute of Technology, Coimbatore, India \\ Email: "leena_eee2002@yahoo.co.in,b.doraarulselvi@gmail.com, lalrajasingh@yahoo.com
}

Received 1 April 2016; accepted 1 May 2016; published 19 July 2016

Copyright (C) 2016 by authors and Scientific Research Publishing Inc.

This work is licensed under the Creative Commons Attribution International License (CC BY).

http://creativecommons.org/licenses/by/4.0/

(c) (i) Open Access

\section{Abstract}

The electric power generation system has always the significant location in the power system, and it should have an efficient and economic operation. This consists of the generating unit's allocation with minimum fuel cost and also considers the emission cost. In this paper we have intended to propose a hybrid technique to optimize the economic and emission dispatch problem in power system. The hybrid technique is used to minimize the cost function of generating units and emission cost by balancing the total load demand and to decrease the power loss. This proposed technique employs Particle Swarm Optimization (PSO) and Neural Network (NN). PSO is one of the computational techniques that use a searching process to obtain an optimal solution and neural network is used to predict the load demand. Prior to performing this, the neural network training method is used to train all the generating power with respect to the load demand. The economic and emission dispatch problem will be solved by the optimized generating power and predicted load demand. The proposed hybrid intelligent technique is implemented in MATLAB platform and its performance is evaluated.

\section{Keywords}

Particle Swarm Optimization (PSO), Economic Dispatch (ED), Economic Dispatch Problems (EDPs), Genetic Algorithm (GA), Neural Network (NN)

\section{Introduction}

An electric power system is defined in the I.E.E. Regulation as a complex interconnection of simple electric

${ }^{*}$ Corresponding author.

How to cite this paper: Rose, R.L., Selvi, B.D.A. and Singh, R.L.R. (2016) Development of Hybrid Algorithm Based on PSO and NN to Solve Economic Emission Dispatch Problem. Circuits and Systems, 7, 2323-2331.

http://dx.doi.org/10.4236/cs.2016.79202 
devices (represented by active and passive elements) in which there is at least one closed path for the flow of current [1]. A power system consists of components such as generators, lines, transformers, loads, switches and compensators [3] [4]. Electric power systems can be divided into two sub-systems, namely, transmission systems and distribution systems [2]. The main process of a transmission system is to transfer electric power from electric generators to the customer area, whereas a distribution system provides an ultimate link between high voltage transmission systems and consumer service [5]. In an electric power system operation, the objective is to achieve the most economical generation policy that could supply the local load demands without violating constraints [6] [7].

Traditionally, economic dispatch (ED) plays a significant role in order to allocate a combination of generation levels to the generating units so that the demand system could be comprehensive and most economic [8]. ED is the most important optimization problem in power systems that have the objective of dividing the power demand among the online generators economically while satisfying various constraints [9] [10]. Since the cost of the power generated is exorbitant, optimum dispatch saves a considerable amount of money [11]. Each of the above principles is used with the following constraints such as power balance constraints, generator limit constraints, valve point coefficients and emission constraints [12] [13].

The objective of the Economic Dispatch Problems (EDPs) of electric power generation is to schedule the committed generating unit outputs so as to meet the required load demand at minimum operating cost while satisfying all the units and system equality and inequality constraints [14] [15]. In Power Market, the optimization of economic dispatch is of economic value to the network operator. The economic dispatch is a relevant procedure in the operation of a power system [16]. The target of the EDP is to determine the optimum generation levels of all online generators in order to minimize the total fuel cost without violating the operational constraints [17]. The ED problem has been solved in two stages. In the first stage, the most economic fuel of each unit is identified and in the second stage the Economic Load Dispatch (ELD) is performed for the selected fuels [18] [19]. Therefore, it is claimed that an Economic Load Dispatch function which to minimize the total fuel cost while at thermal power units satisfying the total demand subjected to the operating constraints of a power system [20] [21].

The economic dispatch problems have shown that they solve the problem by utilizing different types of optimization techniques. They have optimized the generator output power in a fixed range and so the basic procedures of optimization method are not scalable for variations of generator power. So the predicted generator output power will be inaccurate. The output of the optimizing techniques depends on the number of generation units. If the numbers of generation units are increased, satisfactory output may not be obtained and the fitness of the output power also gets affected. The affected output leads to power quality issues in the generated power. The power quality problem affects the real power value that leads to changes in the power factor. All these factors increase the power generating cost. Another most important parameter is emission cost. If the emission cost is high, it is considered that the system affects the environment more. In some of the optimization technique settings the initial value is difficult because the initial value has been chosen at random. So, the iterative processes become complex and the final solution requires to be approximated. In literature, though very few works have attempted to solve the economic dispatch problem by considering the generating cost and the emission cost using hybrid optimization techniques. From this, it has been observed that there exists a need for evolving simple and effective methods for obtaining an optimal solution for Economic Emission Dispatch Problem. Hence in this paper, an attempt has been made to hybrid PSO with ANN for obtaining optimum solution for the Economic Emission Dispatch Problem.

In a generation system, economic and emission dispatch problems are the two primary but different problems to be essentially considered. The economic dispatch problem targets to minimize the operating cost or total fuel cost of the system, which may violate the emission limits. The emission dispatched problem targets simply to minimize the emission from the system, which may violate the economic limits. These evaluations are used to determine the optimum combinations i.e., unit commitment of the generating units, which is subjected to minimize the total fuel cost. Therefore, it is necessary to determine the cost factors of both problems, which is analysed in the next section

\section{Problem Formulation}

\subsection{Determination Economic and Emission Dispatch Problem}

Economic dispatch is the method to find the optimum output of the number of generating units, which may meet 
the system demand at the possible lowest cost. The main objective of the economic dispatch problem is to minimize the operating cost or fuel cost. The fuel cost of all the generating units is determined using the equation (1). The entire production cost of the system is the combination of each generating unit fuel cost. The expression of the total production cost is given as,

$$
E C_{T}=\sum_{i=1}^{N_{G}}\left(a_{i} P_{G i}^{2}+b_{i} P_{G i}+c_{i}\right)
$$

where, $N_{G}$ is the total number of generating units, $E C_{T}$ is the total production cost, $P_{i}$ is the power output of the $i^{\text {th }}$ generating unit, $P_{i}^{\text {min }}$ is the minimum output of the $i^{\text {th }}$ generating unit, $a_{i}, b_{i}, c_{i}$ are the fuel cost coefficient of $i^{\text {th }}$ unit. Equation (1) is used to find the total production cost of the generating units. The economic dispatch problem provides the minimum production cost for the required demand. Emission dispatch is another problem; it is mainly considered the fossil-fired pollutant, i.e., total COx emission quantity of each power generation unit. The emission dispatched problem minimizes the emission from the system. The emission dispatched expression is given below.

$$
E M_{T}=\sum_{i=1}^{N_{G}}\left(\alpha_{i} P_{G i}^{2}+\beta P_{G i}+\gamma_{i}\right)
$$

where, $N_{G}$ is the total number of generating units, $\alpha_{c i}, \beta_{c i}$, and $\gamma_{c i}$ are the $C O x$ emission coefficient of $i^{\text {th }}$ generator, $P_{G i}$ is the power generated at the $i^{t h}$ unit. The economic and emission dispatched problem formulation is made to minimize the objective functions simultaneously, i.e., combination of fuel cost or operating cost and $\mathrm{COx}$ emission, while satisfy the equality and inequality constraints. The equality and inequality constraints are mentioned in the Section 2.2. This evaluation is used to find the best unit commitment, with the use of PSO. It generates the random number of generating unit combinations, which satisfying the total demands of the system. Then the NN is trained with the random number of the optimum generation unit combinations. It gives the resultant unit commitment of the appropriate system demand.

\subsection{Equality and Inequality Constraints}

The power generation of the units must meet the total demand and losses of the system. The equality constraint is given by.

$$
\left[\begin{array}{c}
S_{1} \\
S_{2} \\
\vdots \\
S_{N}
\end{array}\right]=\left[\begin{array}{c}
P_{G 1}+j Q_{G 1} \\
P_{G 2}+j Q_{G 2} \\
\vdots \\
P_{G N_{G}}+j Q_{G N_{G}}
\end{array}\right]=\left[\begin{array}{c}
P_{D 1}+j Q_{D 1} \\
P_{D 2}+j Q_{D 2} \\
\vdots \\
P_{D N_{B}}+j Q_{D N_{B}}
\end{array}\right]+\left[\begin{array}{c}
P_{L 1}+j Q_{L 1} \\
P_{L 2}+j Q_{L 2} \\
\vdots \\
P_{L N_{B}}+j Q_{L N_{B}}
\end{array}\right]
$$

where, $P_{G 1}, P_{G 2}, \cdots, P_{G N_{G}}$ and $Q_{G 1}, Q_{G 2}, \cdots, Q_{G N_{G}}$ is the real and reactive power generated in the $1,2, \cdots, N_{G}$ units, $P_{D 1}, P_{D 2}, \cdots, P_{D N_{B}}$ and $Q_{D 1}, Q_{D 2}, \cdots, Q_{D N_{B}}$ is the active and reactive power demand in the $1,2, \cdots, N_{B}$ buses, $P_{L 1}, P_{L 2}, \cdots, P_{L N_{B}}$ and $Q_{L 1}, Q_{L 2}, \cdots, Q_{L N_{B}}$ is the real and reactive power losses in the $1,2, \cdots, N_{B}$ buses. Each generating unit is operated by its real and reactive power output limits, which provide the stable working condition. The inequality real and reactive power constraints are given by.

$$
\begin{aligned}
& P_{G i}^{\min } \leq P_{G i} \leq P_{G i}^{\max } ; i=1,2, \cdots, N_{G} \\
& Q_{G i}^{\min } \leq Q_{G i} \leq Q_{G i}^{\max } ; i=1,2, \cdots, N_{G}
\end{aligned}
$$

$P_{G i}^{\min }$ and $Q_{G i}^{\min }$ are the minimum values of real and reactive power output of $i^{\text {th }}$ generating unit, $P_{G i}^{\max }$ and $Q_{G i}^{\max }$ are the maximum values of real and reactive power output of $i^{\text {th }}$ generating unit. The generating units are operated at the minimum to a maximum range, which is given in the equations (4) and (5). The limited condition provides the stable operation of the generating units. The transmission loss of the system can be calculated by the following Equations (6) and (7).

$$
P_{L}=\sum_{i=1}^{N_{b}} \sum_{j=1}^{N_{b}}\left[A_{i j}\left(P_{i} P_{j}+Q_{i} Q_{j}\right)+B_{i j}\left(Q_{i} P_{j}-P_{i} Q_{j}\right)\right]
$$




$$
Q_{L}=\sum_{i=1}^{N_{b}} \sum_{j=1}^{N_{b}}\left[C_{i j}\left(P_{i} P_{j}+Q_{i} Q_{j}\right)+D_{i j}\left(Q_{i} P_{j}-P_{i} Q_{j}\right)\right]
$$

With $A_{i j}, B_{i j}, C_{i j}$ and $D_{i j}$ are the loss coefficients, $\delta_{i}$ and $\delta_{j}$ are load angles at the buses, $R_{i j}$ and $X_{i j}$ and are the real and reactive components of the impedance bus matrix. The economic and emission dispatched problem is converted into a single problem, i.e., price penalty factor. The price penalty factor is examined in the following Section 2.3.

\subsection{Price Penalty Factor Calculation}

The Price Penalty Factor consists of both the economic and emission dispatched problems, which is shown in the equation (8). Depending on this factor the rate of the penalty price is fixed. It is described as follows,

$$
F=\frac{E_{C T}\left(P_{i}^{\max }\right) / P_{i}^{\max }}{E_{M T}\left(P_{j}^{\max }\right) / P_{j}^{\max }}
$$

where, $F$ is the price penalty factor, $i$ is the highest fuel cost unit, $j$ is the highest pollutant emission unit. In this proposed work, the combined objective function is described by

$$
\text { Min } \Phi_{T}=\left[w_{E C} * E_{C T}+F * w_{E M} * E_{M T}\right]
$$

where, $\Phi_{T}$ is the combined objective function, $w_{E C}$ and $w_{E M}$ are the weighting factors of economic dispatch and emission dispatch problem. The two weighting factors are provided in many ways. In the classical economic dispatch problem, both weighting factors are $w_{E C}=1.0$ and $w_{E M}=0$. In the pure economic dispatch problem, the resulted weighting factors are $w_{E C}=0$ and $w_{E M}=0.1$. In combined economic and emission problem, the two weighting factors should be equal. The weighting factors of the proposed technique are chosen as 0.5 . The proposed technique is used to determine the unit commitment of the generating units. The generating power of each unit can be determined by the following equation (10)

$$
=\frac{1-\frac{\left(b_{i}+F_{m} \tau_{i}\right)}{\lambda_{u, v}}-\sum_{\substack{i=1 \\ j \neq i}}^{n} 2 B_{i j} P_{j}}{\frac{\left(2 a_{i}+2 F_{m} a_{i}\right)}{\lambda_{u, v}}+2 B_{i i}}
$$

where, $P_{i}$ is power generation of the $i^{\text {th }}$ unit, $\lambda_{u, v}=\lambda_{\min }+\lambda_{N o r}\left(\lambda_{\max }-\lambda_{\min }\right)$ is the actual incremental cost, let $\lambda_{\min }$ and $\lambda_{\max }$ minimum and maximum values of system incremental cost, $\lambda_{\text {Nor }}$ normalized system incremental cost, $F_{m}$ is the price penalty factor it is shown in the equation (9), $a_{i}, b_{i}$ and $\tau_{i}$ is the fuel cost and emission coefficients. The objective functions are used to find the low cost combinations among the given generating units and satisfying the demand. The optimum combination of the generating unit is calculated with the use of the hybrid technique i.e., PSO and NN. The PSO technique is used to generate the random numbers of generating units, which satisfying the system demands with minimized objective function. The hybrid technique contains another part that is NN, which reduces the time of the system evaluations. The proposed system the NN has been trained by the optimum combinations of generating units for every demand. It takes very less time to the evaluations.

\section{Proposed Hybrid Technique Based on PSO and NN}

The Proposed Hybrid Technique is the combination of PSO and NN methods. It is used to reduce the evaluation time and the resultant is very accurate due to the tuning process. The PSO Technique is used to generate the random number of the combinations i.e., the best unit commitment for each demand, which is used to train the neural network. The neural network consists of two inputs i.e., current demand of the system and the previous demand of the system, that has the $N$ number of outputs. The PSO technique is analyzed in the next section.

\subsection{Determination of Optimum Combination of Generating Units Using Particle Swarm Optimization}

PSO is a multi-agent search technique that traces its evolution to the emergent motion of a flock of birds 
searching for food. It has quick convergence speed and optimal searching ability for solving large-scale optimization problems. It was developed by James Kennedy and Russel Eberhart in 1995. In a PSO system, particles fly around in a multidimensional search space. During flight, each particle adjusts its position according to its own experience, and the experience of neighbouring particles, making use of the best position encountered by itself and its neighbours [22] [23]. The best position of the particle and its previous position is represented as $P_{\text {best }}$. The best position among the group is known as the $G_{\text {best }}$, which is used to find the optimum combination of the generating units. The proposed technique PSO is used to find the unit commitment, which minimizes the objective function. The procedures to find the optimum generating units are given as follows.

Step 1: Initializes all the generation unit values.

Step 2: Randomly generates the combination of the generating units.

Step3: Evaluates the combination of the objective function, i.e., the combination of the economic and emission dispatch fuel costs. The generated values must satisfy the condition given below.

$$
\frac{P_{G}-P_{D}}{P_{G}} \leq 0.1
$$

Step 4: Sets the iteration count $i=1$

Step 5: Finds the initial velocity of each particle between the intervals $\left(-V_{\max }, V_{\max }\right)$

Step 6: Selects the new particle to update the velocity of each particle.

Step 7: Evaluates the new particles and select the $P_{\text {best }}=$ best (new) and update the $G_{\text {best }}$.

The process continues until the optimum combination of the generating unit is achieved. When the procedure is completed, the optimum combinations of the generating units are determined. Once the procedure is completed, the unit commitment for every demand is used for the training of the NN.

$\mathrm{NN}$ is an excellent technique to develop the mathematical structures with the ability to learn. It has the capacity to extract the meaning from the complicated data. It has many advantages, such as nonlinearity, mapping input signals to desired response, adaptivity and evidential response. In the proposed technique, the PSO optimum combination results are used to train the neural network, which gives optimum results in very less time. NN has two stages i.e., training and testing stage.

The training of the neural network and the weight adjustments of the neuron is achieved by using the back propagation algorithm.

Step1: Initializes the weights of all the neurons,

Step2: Training datasets are applied to the NN so as to determine the BP error.

Step3: The network output can be calculated.

Step4: Adjusts the weights of all the neurons as $z=z+\Delta z$, where, $z$ is the weight of the neuron, $\Delta z$ is the change in weight of the neuron, which can be determined by,

$$
\Delta z=\beta * U C_{O} * B_{E}
$$

Step 5: Repeats the process from step 2, until the back propagation error to a least value. Once the process gets completed, the network is ready to provide unit commitment output. The results minimize the objective function. The fine tuning process is illustrated in the following Section 3.2.

\subsection{Fine Tuning}

The fine tuning process is performed after the output of the NN, which is used to reorganize the generation units minimizes the objective function. Then the fitness evaluations are determined and analyses the objective function. Figure 1 shows the proposed technique working structure. The overall process provides the required minimized objective functions. This process is implemented in the MATLAB platform, which evaluates the objective function for every power demand. The fuel cost and emission cost for every demand has been calculated. The Proposed Hybrid Technique results are compared with the existing methods.

\section{Experimental Results and Discussions}

The Proposed Hybrid Technique is implemented in the MATLAB platform, which is a combination of the PSO Method and the NN Method. In PSO technique random number of generating units are selected depending on 
the demand, which finds the best combination among the given combinations. The optimum combination of the generating units are determined by the minimized objective function. Then the second step the NN is used to produce better unit commitment results for the every demand. Already the NN trained by the power demand with the corresponding unit commitment. The results are accurate and efficient in the proposed method. Here the performance of the Proposed Hybrid Technique is compared with the existing GA method. The performance of the Proposed Hybrid Method is analyzed with every demand for IEEE 30 bus system.

For every demand, the total cost of the system is identified, which is determined by both the techniques of hybrid method and GA. The results of the both hybrid method and the GA method is given in Table 2.

Table 1 shows the performance of the Proposed Hybrid Method. Its comparative performance with GA is given in Table 2 and the graphical representation of the comparison is given in Figure 2.

Table 2 illustrates the performance analysis of the existing GA method and the Proposed Hybrid Method. In this analysis the power demand is given as follows $150 \mathrm{MW}, 180 \mathrm{MW}, 200 \mathrm{MW}, 230 \mathrm{MW}, 250 \mathrm{MW}, 260 \mathrm{MW}$, $300 \mathrm{MW}, 340 \mathrm{MW}, 350 \mathrm{MW}$ and $400 \mathrm{MW}$. The corresponding total cost using the existing GA method is as

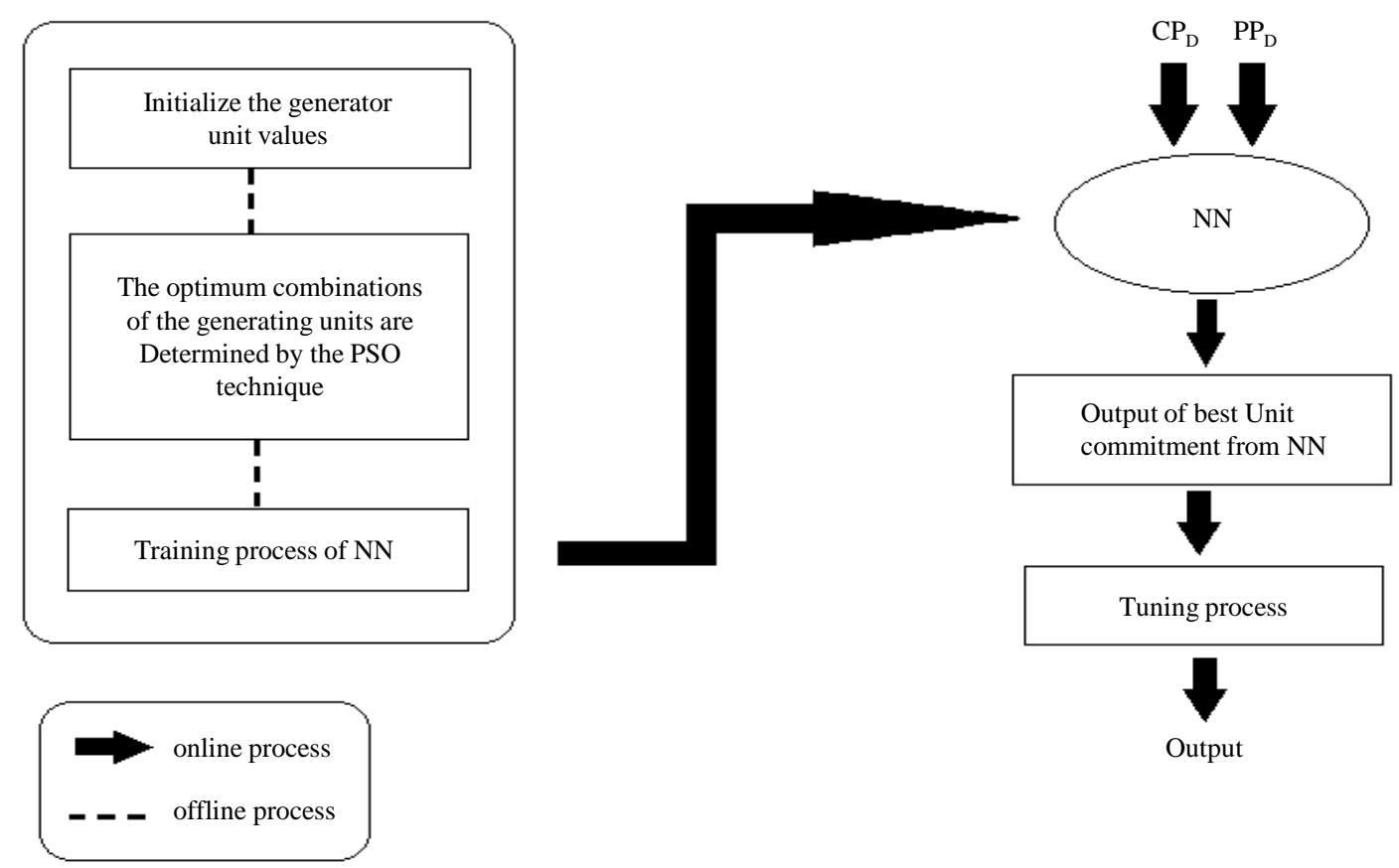

Figure 1. Hybrid technique structure.

Table 1. Total cost of the proposed hybrid technique in IEEE 30 bus system.

\begin{tabular}{|c|c|c|c|c|c|}
\hline \multirow{2}{*}{ Sl. No } & Power Demand & Fuel Cost & Emission Cost & Total Cost Before Tuning & Total Cost After Tuning \\
\hline & MW & $\$ / \mathrm{hr}$ & $\$ / h r$ & $\$ / \mathrm{hr}$ & $\$ / h r$ \\
\hline 1 & 150 & 383.646 & 171.065 & 554.5943 & 459.7613 \\
\hline 2 & 180 & 461.7312 & 198.496 & 553.7204 & 548.0276 \\
\hline 3 & 200 & 429.7505 & 190.1615 & 386.5604 & 377.6988 \\
\hline 4 & 230 & 632.8212 & 315.6549 & 703.6968 & 703.5854 \\
\hline 5 & 250 & 714.6709 & 349.5866 & 744.2272 & 685.5941 \\
\hline 6 & 260 & 723.8457 & 371.5646 & 761.4799 & 736.6453 \\
\hline 7 & 300 & 893.6805 & 514.8531 & 865.5629 & 851.3701 \\
\hline 8 & 340 & 939.4938 & 496.2872 & 939.5361 & 909.1563 \\
\hline 9 & 350 & 1148.3271 & 701.2115 & 1078.706 & 1051.790 \\
\hline 10 & 400 & 1057.8796 & 671.5002 & 1078.8 & 1077.110 \\
\hline
\end{tabular}


Table 2. Performance comparison of GA and hybrid method for IEEE 30 bus system.

\begin{tabular}{cccc}
\hline \multirow{2}{*}{ SI.No } & Power demand & Total cost using GA method & Total cost using hybrid method \\
\cline { 2 - 3 } 1 & MW & $\$ / \mathrm{hr}$ & \$/hr \\
2 & 150 & 513.9195 & 559.7613 \\
3 & 180 & 801.8116 & 548.0276 \\
4 & 200 & 771.6955 & 377.6988 \\
5 & 230 & 711.1035 & 703.5854 \\
6 & 250 & 755.7312 & 685.5941 \\
7 & 260 & 801.1265 & 736.6453 \\
8 & 300 & 858.1526 & 851.3701 \\
9 & 340 & 981.0335 & 909.1563 \\
10 & 350 & 1298.1529 & 1051.7908 \\
\hline
\end{tabular}

\section{PERFORMANCE ANALYSIS}

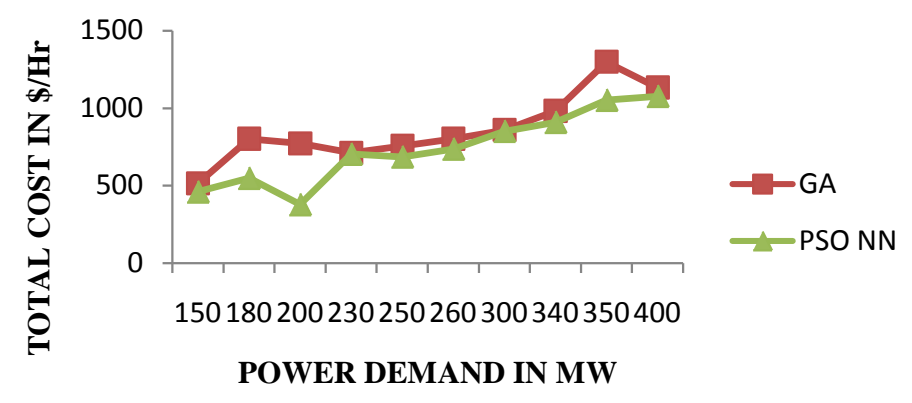

Figure 2. Performance analysis.

Table 3. Performance comparison of different methods for IEEE 30 bus system.

\begin{tabular}{cc}
\hline Method & Total Cost \$/hr \\
\hline Classical Technique [24] & 1247.5 \\
Quadratic Programming [25] & 1252.9 \\
Evolutionary Programming (EPCEED) [26] & 1246.7 \\
Genetic Algorithm & 1132.032 \\
Proposed PSO ANN Hybrid Approach & 1077.1109 \\
\hline
\end{tabular}

follows 513.9195, 801.8116, 771.6955, 711.1035, 755.7312, 801.1265, 858.1526, 981.0335, 1298.1529 and 1132.032 (all in $\$ / h r$ ) respectively. The comparison between the two methods proven that the Proposed Hybrid Method is efficient method as it contains minimized fitness function and it reduces the total cost of the power system.

Table 3 illustrates the performance analysis of the existing methods and the Proposed Hybrid Method. It is thus inferred that there is a reduction in fuel cost using Proposed PSO-ANN Hybrid Approach.

\section{Conclusion}

In this paper we have proposed the hybrid method for optimizing the Economic and Emission dispatch problem in the power system. The proposed hybrid method is the combination of the PSO and NN methods. The problem has been treated as a multi-objective model and the hybrid technique intends to solve the problem. PSO is one of the computational techniques that use a searching process to obtain an optimal solution and neural network is used to predict the load demand. The condition for choosing random generating power value is to satisfy the 
load demand of the distribution system. By using PSO algorithm, the generating power optimized for the given is load demand and at generating cost. The proposed method effectiveness is tested by comparing it with the existing techniques. The comparison results prove the superiority of the proposed hybrid PSO-NN method.

\section{References}

[1] Adedokun, G. (2010) Development of Electrical Power System Simulator. The Pacific Journal of Science and Technology, 11, 144-119.

[2] Thiyagarajan, V. and Palanivel, T.G. (2010) An Efficient Monitoring Of Substations Using Microcontroller Based Monitoring System. IJRRAS, 4, 63-68.

[3] Trar, K. and Soe, T. (2008) Design and Economics of Reactive Power Control in Distribution Substation. World Academy of Science, Engineering and Technology, 48, 416-421.

[4] Molina, M.G. and Mercado, P.E. (2004) Controlling a Static Synchronous Compensator with Superconducting Magnetic Energy Storage for Applications on Primary Frequency Control. Latin American Applied Research, 34, 119-126.

[5] Ilic, M.M., Arce, J.R., Yoon, Y.T. and Fumagalli, E.M. (2001) Assessing Reliability as the Electric Power Industry Restructures. The Electricity Journal, 14, 55-67. http://dx.doi.org/10.1016/S1040-6190(01)00170-1

[6] Senthil, K. and Manikandan, K. (2010) Economic Thermal Power Dispatch with Emission Constraint and Valve Point Effect Loading Using Improved Tabu Search Algorithm. International Journal of Computer Applications, 3, 6-11. http://dx.doi.org/10.5120/770-1080

[7] Manoharan, P.S., Kannan, P.S. and Ramanathan, V. (2009) A Novel EP Approach for Multi-Area Economic Dispatch with Multiple Fuel Options. Turkish Journal of Electrical Engineering and Computer Sciences, 17, 1-19.

[8] Hassan, E.E., Zakaria, Z. and Rahman, T.K.A. (2012) Improved Adaptive Tumbling Bacterial Foraging Optimization (ATBFO) for Emission Constrained Economic Dispatch Problem. Proceedings of the World Congress on Engineering, 2, 1-4.

[9] Kondalu, M., Sreekanth Reddy, G. and Amarnath, J. (2010) A Modified Particle Swarm Optimization to Solve the Economic Dispatch Problem of Thermal Generators of A Power System. International Journal of Engineering Science and Technology, 2, 6140-6148.

[10] Selvakumar, A.I. and Thanushkodi, K., (2007) A New Particle Swarm Optimization Solution to Nonconvex Economic Dispatch Problems. IEEE Transactions on Power Systems, 22, 42-51. http://dx.doi.org/10.1109/TPWRS.2006.889132

[11] Jafari, S., Abdolmohammadi, H.R., Nazari, M.E. and Shayanfar, H.A. (2008) A New Approach for Global Optimization in High Dimension Problems. IEEE Power and Energy Society General Meeting-Conversion and Delivery of Electrical Energy, Pittsburgh, PA, 20-24 July 2008, 1-7. http://dx.doi.org/10.1109/PES.2008.4596830

[12] Abido, M.A. (2003) Environmental/Economic Power Dispatch Using Multiobjective Evolutionary Algorithms. IEEE Transactions on Power Systems, 18, 1529-1537. http://dx.doi.org/10.1109/TPWRS.2003.818693

[13] dos Santos Coelhoa, L. and Leeb, C.-S. (2008) Solving Economic Load Dispatch Problems in Power Systems Using Chaotic and Gaussian Particle Swarm Optimization Approaches. International Journal of Electrical Power \& Energy Systems, 30, 297-307. http://dx.doi.org/10.1016/j.ijepes.2007.08.001

[14] dos Santos Coelho, L. and Mariani, V.C. (2006) Combining of Chaotic Differential Evolution and Quadratic Programming for Economic Dispatch Optimization with Valve-Point Effect. IEEE Transactions on Power Systems, 21, 989996. http://dx.doi.org/10.1109/TPWRS.2006.873410

[15] dos Santos Coelhoa, L. and Marianib, V.C. (2007) Improved Differential Evolution Algorithms for Handling Economic Dispatch Optimization with Generator Constraints. Energy Conversion and Management, 48, 1631-1639. http://dx.doi.org/10.1016/j.enconman.2006.11.007

[16] David, A.K. and Wen, F.S. (2000) Strategic Bidding in Competitive Electricity Markets: A Literature Survey. IEEE Power Engineering Society Summer Meeting, 4, 2168-2173. http://dx.doi.org/10.1109/pess.2000.866982

[17] Abdelaziza, Y., Mekhamera, S.F., Badra, M.A.L. and Kamha, M.Z. (2008) Economic Dispatch Using an Enhanced Hopfield Neural Network. Electric Power Components and Systems, 36, 719-732. http://dx.doi.org/10.1080/15325000701881969

[18] Subramanian, S. and Ganesan, S. (2010) A Simplified Approach for Economic Dispatch with Piecewise Quadratic Cost Functions. International Journal of Computer and Electrical Engineering, 2, 793-798. http://dx.doi.org/10.7763/IJCEE.2010.V2.230

[19] Srinivasa Rao, C., Siva Nagaraju, S., Sangameswara Raju, P. and Rajender Reddy, M. (2007) Optimized Integral Controller For Economic Load Dispatch in a Two Area System Based On Hooke-Jeeves Algorithm. ARPN Journal of Engineering and Applied Sciences, 2, 49-56. 
[20] Yalcinoz, T. and Yalcinoz, T. (2002) Environmentally Constrained Economic Dispatch via a Genetic Algorithm with Arithmetic Crossover. IEEE AFRICON. 6thAfricon Conference in Africa, 2, 923- 928. http://dx.doi.org/10.1109/AFRCON.2002.1160037

[21] Chowdhury, B.H. and Rahman, S. (1990) A Review of Recent Advances in Economic Dispatch. IEEE Transactions on Power Systems, 5, 1248-1259. http://dx.doi.org/10.1109/59.99376

[22] Khamsawang, S. and Jiriwibhakorn, S. (2009) Solving the Economic Dispatch Problem by Using Differential Evolution. International Journal of Energy and Power Engineering, 2, 120-124. http://dx.doi.org/10.1109/ecticon.2009.5136995

[23] Soni, S.K. and Bhuria, V. (2012) Multi-Objective Emission Constrained Economic Power Dispatch Using Differential Evolution Algorithm. International Journal of Engineering and Innovative Technology, 2, 120-125.

[24] Gupta, A., Swarnkar, K.K., Wadhwani, S. and Wadhwani, A.K. (2012) Combined Economic Emission Dispatch Problem of Thermal Generating Units Using Particle Swarm Optimization. International Journal of Scientific and Research Publications, 49, 1-5.

[25] Nanda, J., Hari, L. and Kothari, M.L. (1994) Economic Emission Load Dispatch With Line Flow Constraints Using a Classical Technique. IEE Proceedings-Generation, Transmission and Distribution, 141, 1-10. http://dx.doi.org/10.1049/ip-gtd:19949770

[26] Hota, P.K., Chakrabarti, R. and Chattopadhayay, P.K. (2000) Economic Emission Load Dispatch With Line Flow Constraints Using Sequential Quadratic Programming Technique. India: Institution of Engineers, 81, 21-25.

\section{Submit or recommend next manuscript to SCIRP and we will provide best service for you:}

Accepting pre-submission inquiries through Email, Facebook, LinkedIn, Twitter, etc. A wide selection of journals (inclusive of 9 subjects, more than 200 journals)

Providing 24-hour high-quality service

User-friendly online submission system

Fair and swift peer-review system

Efficient typesetting and proofreading procedure

Display of the result of downloads and visits, as well as the number of cited articles

Maximum dissemination of your research work

Submit your manuscript at: http://papersubmission.scirp.org/ 